\title{
SUBSPACES ASSOCIATED WITH BOUNDARY POINTS OF THE NUMERICAL RANGE
}

\author{
S. MAJUMDAR and BRAILEY SIMS
}

(Received 25 March 1983)

Communicated by $R$. Vyborny

\begin{abstract}
Stampfli and Embry characterized points in the numerical range which are extreme in terms of the linearity of corresponding sets of vectors. Das and Craven generalized this to include the case of unattained boundary points. We give an alternative proof of this result using a technique of Berberian. This approach appears to be more conceptual in that it enables us to deduce the result from that of Stampfli and Embry. We also illustrate how the same technique may be used to generalize other results of Embry.
\end{abstract}

1980 Mathematics subject classification (Amer. Math. Soc.); 47 A 12.

\section{Introduction}

Let $H$ be a complex Hilbert space with inner product $\langle$,$\rangle and induced norm$ $\|\cdot\|$. The numerical range $W(T)$ of an operator (i.e. a bounded linear transformation) on $H$ is the set

$$
W(T)=\{\langle T x, x\rangle:\|x\|=1, x \in H\} .
$$

The Toeplitz-Hausdorff theorem asserts that $W(T)$ is a convex set in the plane. For a given $z \in \mathbf{C}$, let $M_{z}(T)=\{x:\langle T x, x\rangle-z\langle x, x\rangle=0, x \in H\}$. J. G. Stampfli [1966] and M. R. Embry [1970, 1975] have shown that a point $z$ of the numerical range $W(T)$ is extreme if and only if $M_{z}(T)$ is a subspace. This has been generalized by K. C. Das and B. D. Craven [1983] to show that a point $z$ of $W(T)^{-}$, the closure of $W(T)$, is extreme if and only if

$$
N_{z}(T)=\left\{\left(x_{n}\right):\left\langle T x_{n}, x_{n}\right\rangle-z\left\langle x_{n}, x_{n}\right\rangle \rightarrow 0,\left(x_{n}\right) \in l_{\infty}(H)\right\},
$$

(C) 1985 Australian Mathematical Society $0263-6115 / 85 \$ A 2.00+0.00$ 
is a subspace where $l_{\infty}(H)$ is the set of all bounded sequences of vectors from $H$.

In this note we give an alternative approach to establish the same result employing a technique of S. K. Berberian [1962], and S. K. Berberian and G. H. Orland [1967]. This approach appears to be more conceptual in that it enables us to deduce the result of Das and Craven from that of Stampfli and Embry.

Using the same technique other results may also be generalized to unattained boundary points. This is illustrated in Section 5 where we extend results of Embry [1975].

\section{A technical lemma}

Let $l_{\infty}, l_{\infty}^{+}, c$ and $c_{0}$ be the sets of real bounded, bounded nonnegative, convergent and null sequences respectively. Let $x=\left(x_{n}\right)=\left(x_{1}, x_{2}, \ldots, x_{n}\right) \in l_{\infty}$ and $l_{\infty}^{*}$ be the dual of $l_{\infty}$.

We prove a simple lemma which will be used in the following sections to prove our main results.

LEMMA 1. For any element $y \in l_{\infty}^{+} \backslash c_{0}$, there exists $f \in l_{\infty}^{*}$ such that

(i) $f(y)>0$,

(ii) $f$ is positive, that is, $f(x) \geqslant 0$ for all $x \in l_{\infty}^{+}$,

(iii) $f(e)=1$ where $e=(1,1, \ldots)$ and so $\|f\|=1$,

(iv) $\left.f\right|_{c_{0}}=0$, and

(v) For all $x \in l_{\infty}, \lim \inf x_{n} \leqslant f(x) \leqslant \lim \sup x_{n}$, in particular, for $x \in c$, $f(x)=\lim x_{n}$.

In other words, $y$ may be strictly separated from $c_{0}$ by a 'normalized positive linear functional'.

Proof. Let $A=\left\{x \in l_{\infty}: \lim \sup x_{n} \leqslant 0\right\}$. It can be verified that $A=c_{0}-l_{\infty}^{+}$ and that $A$ is closed and convex. Obviously $y \notin A$ since $y \in l_{\infty}^{+} \backslash c_{0}$. Hence there exists $g \in l_{\infty}^{*}$ with $g(y)>0=\sup g(A)$. If $x \in c_{0}$ then $x,-x \in c_{0} \subseteq A$, so we have both $g(x)$ and $g(-x) \leqslant 0$ and $g(x)=0$. Also, $x \in l_{\infty}^{+}$implies $-x \in A$. So $g(-x) \leqslant 0$, or $g(x)>0$; that is $g$ is positive on $l_{\infty}^{+}$. Further $\|y\| e-y \in l_{\infty}^{+}$, so $g(\|y\| e-y) \geqslant 0$ and $g(e) \geqslant 0$. Write $f=g / g(e)$, then $f$ satisfies (i) to (iv) and therefore (v) which is an immediate consequence of (ii), (iii) and (iv). 


\section{A modification of Berberian's technique}

S. K. Berberian [1962] used the existence of a Banach-Mazur generalized limit, glim, for bounded sequences of real numbers to introduce a pseudo-inner product on $l_{\infty}(H)$ and thereby obtained a Hilbert space extention $K$ of $H$. In fact glim was only required to be an element of $l_{\infty}^{*}$ satisfying the properties (ii) to (v) of Section 2. Thus, for every $f$ of the type described by Lemma 1 we have the following construction (for details see, Berberian [1962]).

Suppose $s=\left(x_{n}\right)$ and $t=\left(y_{n}\right)$ belong to $l_{\infty}(H)$. Define $\phi(s, t)=$ $f\left(\left(\operatorname{Re}\left\langle x_{n}, y_{n}\right\rangle\right)\right)+i f\left(\left(\operatorname{Im}\left\langle x_{n}, y_{n}\right\rangle\right)\right)$, then $\phi(s, t)$ is a pseudo-inner product on $l_{\infty}(H) . \mathscr{N}=\left\{s \in l_{\infty}(H): \phi(s, s)=0\right\}=\left\{s \in l_{\infty}(H): \phi(s, t)=0\right.$ for all $t \in$ $\left.l_{\infty}(H)\right\}$ is a subspace of $l_{\infty}(H)$, so we can define the quotient inner-product space $P=l_{\infty}(H) / \mathscr{N}$ with inner product $\left\langle s^{\prime}, t^{\prime}\right\rangle=\phi(s, t)$ where $s^{\prime}=s+\mathscr{N}$. Let $K$ be the Hilbert space completion of $P$.

Every operator $T$ in $H$ determines an operator in $K$ as follows. Define $T_{0}$ : $l_{\infty}(H) \rightarrow l_{\infty}(H)$ by $T_{0} s=\left(T x_{n}\right)$ and $T^{0}: P \rightarrow P$ by $T^{0} s^{\prime}=\left(T_{0} s\right)^{\prime}$. The continuous linear mapping $T^{0}$ extends to a unique operator in $K$ which we also denote by $T^{0}$. Berberian and Orland [1967] have shown in the proposition of Section 3 that $W\left(T^{0}\right)=W(T)^{-}$. This fact is basic to our proofs.

\section{Linearity of $N_{z}(T)$}

We are now ready to prove

THEOREM 2. $N_{z}(T)$ is a subspace of $l_{\infty}(H)$ if and only if $z$ is an extreme point of $W(T)^{-}$.

Proof. By carrying out the standard reduction $T \rightarrow \exp (i \theta)(T-z I)$ where $\theta$ is a suitably chosen real number, we can assume without loss of generality that $z=0$ and $\operatorname{Re} W(T) \geqslant 0$. We first prove sufficiency.

Homogeneity being obvious, we need prove only additivity of $N_{z}(T)$.

By the construction of Section 3, for each $f$ of the type described in Lemma 1 we have $W\left(T^{0}\right)=W(T)^{-}$. Indeed if $\left\langle T x_{n}, x_{n}\right\rangle \rightarrow 0$ then $0=\left\langle T^{0} s^{\prime}, s^{\prime}\right\rangle$ where $s^{\prime}=s+\mathscr{N}, s=\left(x_{n}\right)$.

Now let $\left(x_{n}\right),\left(y_{n}\right)$ be such that both $\left\langle T x_{n}, x_{n}\right\rangle$ and $\left\langle T y_{n}, y_{n}\right\rangle \rightarrow 0$ (an extreme point of $\left.W(T)^{-}\right)$. Then $\left\langle T^{0} s^{\prime}, s^{\prime}\right\rangle=\left\langle T^{0} t^{\prime}, t^{\prime}\right\rangle=0$ is an extreme point of 
$W\left(T^{0}\right)$, so by Theorem 1 of Embry [1970], $\left\langle T^{0}\left(s^{\prime}+t^{\prime}\right), s^{\prime}+t^{\prime}\right\rangle=0$, or, $\left\langle T\left(\left(x_{n}+y_{n}\right)\right)^{\prime},\left(x_{n}+y_{n}\right)^{\prime}\right\rangle=0$. Thus, by the form of the inner-product in $K$ we have for each $f$ that

$$
\begin{aligned}
& f\left(\left(\operatorname{Re}\left\langle T\left(x_{n}+y_{n}\right), x_{n}+y_{n}\right\rangle\right)\right)=0, \\
& f\left(\left(\operatorname{Im}\left\langle T\left(x_{n}+y_{n}\right), x_{n}+y_{n}\right\rangle\right)\right)=0 .
\end{aligned}
$$

Let $\alpha=\left(\alpha_{n}\right)$ and $\beta=\left(\beta_{n}\right)$.

Now, $\alpha=\left(\operatorname{Re}\left\langle T\left(x_{n}+y_{n}\right), x_{n}+y_{n}\right\rangle\right) \in l_{\infty}^{+}$and so by (1) and Lemma 1, $\alpha \in c_{0}$ or $\alpha_{n} \rightarrow 0$. To show $\beta_{n}=\operatorname{Im}\left\langle T\left(x_{n}+y_{n}\right), x_{n}+y_{n}\right\rangle \rightarrow 0$ requires a little more work. First note that

$$
\lim \inf \beta_{n} \leqslant f(\beta) \leqslant \lim \sup \beta_{n} .
$$

Also, by (2), $f(\beta)=0$. Assume $a=\lim \sup \beta_{n}>0$, then there exists a subsequence $\left(n_{k}\right)$ such that

$$
\operatorname{Im}\left\langle T\left(x_{n_{k}}+y_{n_{k}}\right), x_{n_{k}}+y_{n_{k}}\right\rangle \rightarrow a .
$$

Passing on to a further subsequence we may assume

$$
\left\|x_{n_{k}}+y_{n_{k}}\right\| \rightarrow L \neq 0 .
$$

(If $L=0$, then $\beta_{n_{k}} \rightarrow 0$ contradicting $a>0$.) Thus

$$
\operatorname{Im}\left(\frac{T\left(x_{n_{k}}+y_{n_{k}}\right)}{\left\|x_{n_{k}}+y_{n_{k}}\right\|}, \frac{x_{n_{k}}+y_{n_{k}}}{\left\|x_{n_{k}}+y_{n_{k}}\right\|}\right) \rightarrow \frac{a}{L^{2}}
$$

while

$$
\operatorname{Re}\left\langle\frac{T\left(x_{n_{k}}+y_{n_{k}}\right)}{\left\|x_{n_{k}}+y_{n_{k}}\right\|}, \frac{x_{n_{k}}+y_{n_{k}}}{\left\|x_{n_{k}}+y_{n_{k}}\right\|}\right\rangle \rightarrow 0 .
$$

So $i a / L^{2} \in W(T)^{-}$. If also $b=\lim \inf \beta_{n}<0$, we would similarly have $i b / l^{2} \in$ $W(T)^{-}$, where $b / l^{2}<0<a / L^{2}$ contradicting that 0 is an extreme point of $W(T)^{-}$. Thus at least one of $a$ and $b$ is zero. Now $\beta$ can be decomposed as $\beta=\beta^{0}+\left(\beta-\beta^{0}\right)$ where

$$
\beta_{m}^{0}= \begin{cases}\beta_{m}-a, & \text { if } \beta_{m} \geqslant a ; \\ 0 & \text { otherwise. }\end{cases}
$$

So $\beta^{0} \in c_{0}$ and $a e-\left(\beta-\beta^{0}\right) \in l_{\infty}^{+}$. If $a=0, \beta-\beta^{0} \in l_{\infty}^{-}$. Similarly, if $b=0$, $\beta-\beta^{0} \in l_{\infty}^{+}$. But then for all $f$ satisfying the condition of Lemma 1 we have $0=f(\beta)=f\left(\beta-\beta^{0}\right)$ and so $\beta-\beta^{0} \in c_{0}$. Thus $\beta \in c_{0}$ and consequently $N_{0}(T)$ is linear.

To prove the converse we may assume that 0 is not an interior point of $W(T)$ (for which case the proof follows easily from the result of Embry [1970]), and that 0 lies on the join of $i a$ and $-i b$ where $i a$ and $-i b$ belong to $W(T)^{-}, a, b>0$. We will show that $N_{0}(T)$ is not linear. 
Let $s=\left(x_{n}\right)$ and $t=\left(y_{n}\right)$ be two sequences of unit vectors such that $\left\langle T x_{n}, x_{n}\right\rangle \rightarrow i a$ and $\left\langle T y_{n}, y_{n}\right\rangle \rightarrow-i b$. Then, since $\left\langle\left(T+T^{*}\right) x_{n}, x_{n}\right\rangle \rightarrow 0$ and 0 is an extreme point of $W\left(T+T^{*}\right)$, it is also an approximate eigenvalue of the Hermitian operator $T+T^{*}$. It follows that, for any $\lambda$, a subsequence of $\left(\left\langle\left(T\left(x_{n}+\lambda y_{n}\right), x_{n}+\lambda y_{n}\right)\right\rangle\right)$ is convergent. For the sake of simplicity we shall denote the subsequence by the same symbols.

Now, given any $f$ satisfying the conditions of Lemma 1 , we have $\left\langle T^{0} s^{\prime}, s^{\prime}\right\rangle=i a$ and $\left\langle T^{0} t^{\prime}, t^{\prime}\right\rangle=-i b$ and so by Lemma 1 of Embry [1970] we have

$$
\left\langle T^{0}\left(x_{n}+\lambda y_{n}\right)^{\prime},\left(x_{n}+\lambda y_{n}\right)^{\prime}\right\rangle=0
$$

for two distinct values of $\lambda$. By (v) in Lemma 1 and the construction of $K, T^{0}$ we therefore have for both these values of $\lambda$ that

$$
\begin{aligned}
& \lim \left\langle T\left(x_{n}+\lambda y_{n}\right), x_{n}+\lambda y_{n}\right\rangle \\
& \quad=f\left(\left(\operatorname{Re}\left\langle T\left(x_{n}+\lambda y_{n}\right)\right), x_{n}+\lambda y_{n}\right\rangle\right)+i f\left(\left(\operatorname{Im}\left\langle T\left(x_{n}+\lambda y_{n}\right), x_{n}+\lambda y_{n}\right\rangle\right)\right) \\
& \quad=0,
\end{aligned}
$$

that is, $\left(x_{n}+\lambda y_{n}\right) \in N_{0}(T)$ for two distinct values of $\lambda$. Hence $N_{0}(T)$ is not linear.

\section{Generalization of a Cauchy-Schwartz inequality}

In Theorem 2.2 Embry [1975] gives a version of the Cauchy-Schwartz inequality for the vectors associated with points of $L \cap W(T)$, where $L$ is a line of support for $W(T)$. We translate this into a statement about sequences of vectors associated with points of $L \cap W(T)^{-}$. We then illustrate how other results may be extended to unattained boundary points of $W(T)$ by deriving generalizations for some of the consequences given in Section 2 of Embry [1975].

Throughout let $L$ be a line of support for $W(T)^{-}$and define

$$
N_{L}(T)=\left\{\left(x_{n}\right) \in l_{\infty}(H): \inf _{z \in L}\left|\left\langle T x_{n}, x_{n}\right\rangle-z\left\langle x_{n}, x_{n}\right\rangle\right| \rightarrow 0\right\} .
$$

By a suitable translation and rotation we may assume that $L$ is the imaginary axis and $\operatorname{Re} W(T) \geqslant 0$, in which case we see that $\left(x_{n}\right) \in N_{L}(T)$ if and only if $\operatorname{Re}\left\langle T x_{n}, x_{n}\right\rangle \rightarrow 0$.

Since 0 is an extreme point of $W(\operatorname{Re} T)$ and therefore an approximate eigenvalue it follows that

$$
N_{L}(T)=\left\{\left(x_{n}\right) \in l_{\infty}(H):(\operatorname{Re} T) x_{n} \rightarrow 0\right\}
$$

and so we conclude that $N_{L}(T)$ is a subspace of $l_{\infty}(H)$. If we define $f$ on complex 
sequences by $f\left(\left(\lambda_{n}\right)\right)=f\left(\left(\operatorname{Re} \lambda_{n}\right)\right)+i f\left(\left(\operatorname{Im} \lambda_{n}\right)\right)$, we have

LEMMA 3. Let $f$ satisfy the conditions of Lemma 1 and let $z$ be a point of $L$ such that either $z$ is an extreme point of $W(T)^{-}$or $z \notin W(T)^{-}$. Then for all $\left(x_{n}\right)$, $\left(y_{n}\right) \in N_{L}(T)$

$$
\left|f\left(\left(\left\langle(T-z) x_{n}, y_{n}\right\rangle\right)\right)\right|^{2} \leqslant f\left(\left(\left\langle(T-z) x_{n}, x_{n}\right\rangle\right)\right) f\left(\left(\left\langle y_{n},(T-z) y_{n}\right\rangle\right)\right) .
$$

Proof. As above we may assume that $L$ is the imaginary axis, $\operatorname{Re} W(T) \geqslant 0$ and, by a further translation if necessary, that $z=0$. For the given $f$ let $K$ and $T^{0}$ be as in Section 3 and let $s=\left(x_{n}\right), t=\left(y_{n}\right)$, then

$$
\operatorname{Re}\left\langle T^{0} s^{\prime}, s^{\prime}\right\rangle=f\left(\left(\operatorname{Re}\left\langle T x_{n}, x_{n}\right\rangle\right)\right)=0, \quad \text { as }(\operatorname{Re} T) x_{n} \rightarrow 0 .
$$

Similarly $\operatorname{Re}\left\langle T^{0} t^{\prime}, t^{\prime}\right\rangle=0$. Theorem 2.2 of Embry [1975] therefore applies to give

$$
\left|\left\langle T^{0} s^{\prime}, t^{\prime}\right\rangle\right|^{2} \leqslant\left\langle T^{0} s^{\prime}, s^{\prime}\right\rangle\left\langle t^{\prime}, T^{0} t^{\prime}\right\rangle
$$

or, using the definition of inner-product in $K$, that

$$
\left|f\left(\left(\left\langle T x_{n}, y_{n}\right\rangle\right)\right)\right|^{2} \leqslant f\left(\left(\left\langle T x_{n}, x_{n}\right\rangle\right)\right) f\left(\left(\left\langle y_{n}, T y_{n}\right\rangle\right)\right)
$$

as required.

COROllary 4. If $z$ is an extreme point of $W(T)^{--}$and $L$ is a line of support for $W(T)^{-}$passing through $z$, then

$$
\lim \left\langle(T-z) x_{n}, y_{n}\right\rangle=0
$$

for all $\left(x_{n}\right) \in N_{z}(T)$ and $\left(y_{n}\right) \in N_{L}(T)$.

Proof. Without loss of generality assume $z=0, L$ is the imaginary axis and $\operatorname{Re} W(T) \geqslant 0$.

Assume $\left\langle T x_{n}, y_{n}\right\rangle$ does not converge to 0 , then there exists subsequences $\left(x_{n_{k}}\right)$, $\left(y_{n_{k}}\right)$ such that either the real or imaginary parts of $\left\langle T x_{n_{k}}, Y_{n_{k}}\right\rangle$ form a sequence in $l_{\infty}^{+} \backslash c_{0}$ (or $\left.l_{\infty}^{-} \backslash c_{0}\right)$.

By Lemma 1, there is an $f$ with the stated properties such that $f\left(\left(\left\langle T x_{n_{k}}, y_{n_{k}}\right\rangle\right)\right)$ $\neq 0$. To derive a contradiction we note that $\left(x_{n_{k}}\right) \in N_{z}(T) \subseteq N_{L}(T),\left\langle T x_{n_{k}}, x_{n_{k}}\right\rangle$ $\rightarrow 0$ and $\left(y_{n_{k}}\right) \in N_{L}(T)$, thus by Lemma $3 f\left(\left(\left\langle T x_{n_{k}}, y_{n_{k}}\right\rangle\right)\right)=0$.

COROllary 5. Let $z$ and $L$ be as in Corollary 4 .

If $\left(x_{n}\right) \in N_{z}(T)$ and $\left(T x_{n}\right) \in N_{L}(T)$, then

$$
\lim (T-z) x_{n}=\lim \left(T^{*}-\bar{z}\right) x_{n}=0 .
$$


Proof. Again assume $z=0, L$ is the imaginary axis and $\operatorname{Re} W(T) \geqslant 0$.

Since $\left(x_{n}\right) \in N_{z}(T)$, by definition $\left\langle T x_{n}, x_{n}\right\rangle \rightarrow 0$ and so by Lemma 3 $f\left(\left(\left\langle T x_{n}, y_{n}\right\rangle\right)\right)=0$ for all $\left(y_{n}\right) \in N_{L}(T)$. In particular, taking $y_{n}=T x_{n}$ we have $f\left(\left(\left\|T x_{n}\right\|^{2}\right)\right)=0$. Now $\left(\left\|T x_{n}\right\|^{2}\right)$ is in $l_{\infty}^{+}$, so by Lemma 1 we conclude that $T x_{n} \rightarrow 0$ and, since $(\operatorname{Re} T) x_{n} \rightarrow 0$, that $T^{*} x_{n} \rightarrow 0$.

Similar extensions of results by Stampfli, de Barra and Lin for unattained boundary points will be the subject of a subsequent paper by Das, Majumdar and Sims.

\section{References}

S. K. Berberian (1962), 'Approximate proper vectors', Proc. Amer. Math. Soc., 13, 111-114.

S. K. Berberian and G. H. Orland (1967), 'On the closure of the numerical range of an operator', Proc. Amer. Math. Soc., 18, 499-503.

K. C. Das and B. D. Craven (1983), 'Linearity and weak convergence on the boundary of numerical range', J. A ustral. Math. Soc. (Series A), 35, 221-226.

M. R. Embry (1970), 'The numerical range of an operator', Pacific J. Math., 32, 647-650.

M. R. Embry (1975), 'Orthogonality and the numerical range', J. Math. Soc. Japan, 27, 405-411.

J. G. Stampfli (1966), 'Extreme points of the numerical range of a hyponormal operator', Michigan Math. J., 13, 87-83.

University of New England

Armidale, N.S.W. 2351

Australia
University of New England

Armidale, N.S.W. 2351

Australia

Queen's University

Kingston, Ontario

K7L 3N6 Canada 